\title{
Michel Vovelle, 1789. L'héritage et la mémoire
}

Toulouse, Privat, 2007, 376 p., ISBN 972-2-7089-6870-7, $19 €$.

\section{Guy Lemarchand}

\section{(2) OpenEdition}

Journals

\section{Édition électronique}

URL : https://journals.openedition.org/ahrf/11410

DOI : 10.4000/ahrf.11410

ISSN : 1952-403X

Éditeur :

Armand Colin, Société des études robespierristes

Édition imprimée

Date de publication : 1 mars 2008

Pagination : 211-216

ISSN : 0003-4436

\section{Référence électronique}

Guy Lemarchand, «Michel Vovelle, 1789. L'héritage et la mémoire », Annales historiques de la Révolution française [En ligne], 351 | janvier-mars 2008, mis en ligne le 29 décembre 2009, consulté le 03 août 2021. URL : http://journals.openedition.org/ahrf/11410; DOI : https://doi.org/10.4000/ahrf.11410 
le calvinisme, souvent favorable à la République, bascule dans la contre-révolution, il suggère que l'affaiblissement de la foi réformée au XVIII' siècle fournit une des causes de la Vendée, mais l'on peut douter que des pratiques religicuses routinic̀res, à cause de pasteurs gyrovagues peu présents et pas toujours dignes, puissent être remplacées par un catholicisme ardent. Il s'autorise parfois quelques facilités : Dangirard, inutilement qualifié de "pape »du protestantisme, est «propulsé en plein Bas-Poitou, cette future Vendée que l'histoire révèlera à elle-même » (p. 20), comme si la destinée, voire l'essence de cette région, résidait dans la guerre civile. L'empathie tient en outre lieu de rigueur scientifique : "Tout un monde s'esquisse, dont il faut, pour se l'imaginer concrètement, avoir soi-même un peu goûté de la saveur de telles petites républiques paroissiales rivales et solidaires, fortes en tout cas d'une ruralité incntamée » (p. 42). Le propos est enfin idéologiquement connoté : le Comité clandestin qui dirige la communauté poitevine, comporte 14 anciens dont 6 perpétuels, ce qui réalise "l'idéal de démocratie de compétences cher aux Lumières " (p. 17) ! Le Bas-Poitou est décrit comme un " conservatoire de culture paysanne [...] d'avant l'individualisme moderne et la haine de classe » (p. 42). Lan Il est évidemment une " dérive terroriste de la Révolution" (p. 50).

On prend certes plaisir à ce tableau de la vie familiale modifiée par la mode de la nature, où les mères allaitent leurs petits et les pères s'inquic̀tent de la poussée d'une dent. On remarque la modernité du mémorialiste qui aurait aimé " ouvrir » un enfant hydrocéphale et voir l'État s'impliquer dans la santé publique. À la description de ces fêtes de village qui dégénèrent en batailles entre paroisses rivales, on vérific l'une des conditions de possibilité de l'action contre-révolutionnaire (R. Dupuy). On retrouve le loyalisme monarchique chez ce protestant qui recopie une prière pour Marie-Antoinette enceinte. Ce journal peut émouvoir par les souffrances de son auteur qui reste digne, curieux des autres, volontiers caustique à l'égard des mours des papistes, de leurs curés et de leurs saints, néanmoins très soucieux des lents progrès de la tolérance. Toutefois émaillé de notations triviales - " j'ai fait beaucoup de matières moulées et ayant de la consistance, et cela sans effort et dans douleur " (p. 99), «j'ai beaucoup souffert au fondement » (p. 212), etc... -, la représentativité de ce témoignage qui n'avait certainement pas été conçu pour ĉtre publić, paraît assez étroite et sa publication semble s'adresser en priorité à l'érudition locale.

Anne De Mathan

Michel Vovelle, 1789. L'héritage et la mémoire, Toulouse, Privat, 2007, 376 p., ISBN 972-2-7089-6870-7, $19 €$.

Contrairement à ce que son titre pourrait suggérer, ce livre n'est pas une synthèse d'ensemble sur le déroulement de la Révolution, ni même sur l'année 1789. Le sous-titre en donne exactement le contenu et l'intention de l'auteur. Rassemblant des articles et communications de ces dernières années, inédits ou parus dans les publications d'accès difficile, les coordonnant à partir d'une problématique de fond, il s'agit d'abord d'un ouvrage de réflexion à la lumière de la recherche la plus récente sur les acquis pour la France et le monde immédiats et à terme de la Révolution, séquence d'une dizaine d'années seulement. Michel 
Vovelle aurait pu poursuivre jusqu'à la stabilisation de 1815, tant l'Empire est bien dans la suite et complète la Révolution sur ce plan, mais un tel ajout aurait sans doute alourdi et peut-être dilué l'essentiel. Après ces 200 premières pages du volume, dans le vent du chanticr apparu dans les préoccupations des historiens depuis 1980,150 pages sont consacrées à l'historique à grands traits du traitement et des remaniements qu'a subis l'événement au XIX siècle et surtout dans la seconde moitié du $X^{\mathrm{c}}$ siècle jusqu'à aujourd'hui.

La première partie s'articule autour de deux questions fondamentales et liées entre elles : la Révolution constitue-t-elle un " capital " (moral) à préserver, est-elle un événement décisif pour l'histoire de la France et au-delà, et présentet-elle une rupture ou une continuité avec l'Ancien Régime? Dans cette optique s'impose aussitôt pour l'homme du XXI' siècle l'image de la Déclaration des droits de l'homme et du citoyen. Avec une volonté fortement énoncée d'universalité - les hommes et pas seulement les Français - au nom du droit naturel, engageant le pouvoir officiel, la Constituante bouleverse le fondement habituel de l'autorité suprême, droit divin ou sollicitude humanitaire du prince. Le texte n'est pas qu'une affirmation globale de la liberté, il énonce les libertés concrètes, en particulier la liberté économique et le droit de propriété, bases du système capitaliste futur. Mais il ne dit pas tout et le reste sera à conquérir, en partie, dans les années suivantes. Assez discret sur l'égalité afin de ne pas aller jusqu'à la remise en cause de l'inégalité des fortunes, il est silencieux sur la fraternité qui aura place dans la déclaration de 1793 et la révolution de 1848 . Quant à la nation, la souveraineté nationale et la République, plus que des traités et pamphlets (Sieyès) et de la pensée des députés, clles résultent de la volonté populaire, du mouvement des fédérations à la « journée " du 10 août 1792. Officialisée, la conscience nationale, devenue affective (la patrie) ne verse pas pour autant dans le nationalisme, comme en témoignent les définitions des conditions d'accès à la citoyenneté française de 1791 et 1793 ainsi que l'aide offerte aux pcuples étrangers opprimés. Qu'est-ce qui a pu transformer à plusieurs reprises les intentions généreuses en violence arbitraire et conquêtes et pillages ? Michel Vovelle argumente sur la "théorie des circonstances " et de la double guerre, contre la formidable puissance de la coalition des monarchies et contre les ennemis de l'intérieur hostiles à la Révolution par conviction ou suivant l'opportunité. La phrase de Robespierre bien connue citée page 88 "Voulicz-vous une Révolution sans révolution? " dit tout. Parce qu'une révolution au nom de la justice s'en prend aux dominants et à leurs intérêts, à ceux qui tirent précisément parti de l'injustice, elle ne peut pas espérer que ceux-là demeurent sans réaction. Il n'y a pas de révolution sans contre-révolution. Quoi qu'il y paraisse, la Terreur jusqu'aux lois de prairial représente l'ordre, selon Michel Vovelle, dans la " citadelle assiégée " - la recherche actuelle mesure mieux le poids de la guerre - en maîtrisant relativement une violence venue des pratiques anciennes et qu'on retrouve chez les adversaires aussi bien que chez les partisans de la « patric en danger". Et dans l'atmosphère du temps où les peurs et le surnaturel sont encore présents, née des besoins matériels de finance, la crise religieuse, avec la Constitution civile du clergé a exacerbé les haines, pas seulement en Vendée, en Bretagne ou en Anjou, mais au moins a-t-elle consacré finalement, mieux que la tolérance du XVIII' siècle, la liberté de croyance et constitue-t-elle un premier pas vers la séparation de l'Église et de l'État. 
En même temps qu'un long combat, la Révolution a été un apprentissage de la politique, contrairement à ce que voulaient y voir la Restauration, et avant elle l'abbé Barruel ou le comte de Puisaye définissant les Jacobins comme « [...] des vers sur un cadavre » (p. 111) (1802), présentant ces années comme le produit de l'action d'une minorité de déclassés et d'aigris. Par rapport à l'Ancien Régime, il y a en fait une triple rupture. La plus visible est une explosion de l'expression politique, du tract et des journaux jusqu'aux mouvements et cris de la rue. Les élections aussi deviennent une pratique générale et régulière, du local au national, relativement libres et bien organisées, même sous le Directoire ; seulement pendant longtemps l'historiographie ne s'y était guère intéressée. Et même, pendant un à deux ans, la politique a pu être un sujet partout débattu, à travers le réseau des Sociétés populaires, dont on sait aujourd'hui qu'elles n'ont pas été un corps de discipline militaire attendant les ordres de la société-mère. Le jeu des cartes battues par Michel Vovelle, repris de La découverte de la politique... (1993) qui fit date, montre aussi les héritages de l'Ancien Régime, telle l'influence des batailles de la Réforme puis des troubles de Nîmes d'avril-juin 1790 étudiés par V. Sottocasa (2004) qui ont fait adhérer les protestants du Midi à la Révolution et, contre les marchands soyeux, une partie du Sud-Est populaire passer à la contre-révolution. Et ensuite, malgré les transformations sociales, l'exode rural et l'urbanisation, la carte des camps politiques, droite-gauche, tend à se fixer pour longtemps comme le montre la comparaison entre la géographic électorale de l'élection présidentielle de 1965, celle de la pratique religieuse de 1950 , et celles des référendums de 1793 et 1795 et les votes du Directoire.

Enfin le calendrier passagèrement a été changé et les structures administratives durablement ont été rationalisées. Il s'agissait bien de construire une nation unie et le fédéralisme girondin a été d'autant moins une revendication identitaire provinciale que le nouveau découpage, beaucoup moins géométrique et rigide que ne l'ont dit ses détracteurs, a respecté les paroisses et communautés à travers les communes, et - relativement - les provinces et les pays à travers la départementalisation. Sans doute n'est pas survenue à la suite, dans les pratiques, la mise en place rapide d'un marché national ; il y faudra du temps et la révolution industrielle n'a pas surgi dans les années qui ont suivi 1791 ou 1800 parce que l'appropriation des techniques nouvelles et l'accumulation des capitaux nécessaires ne se réalisent pas à l'échelle du temps court, surtout, ajouterai-je, avec la guerre incessante, qui n'empêche tout de même pas ce que L. Bergeron avait appelé une «première révolution industrielle » et E. Labrousse « la croissance dans la guerre».

Dans cette première partie, trois traits frappent le lecteur. Il y a, bien sûr, le renouvellement et l'élargissement de la recherche depuis 1960 où la Révolution était un des centres d'intérêt majeurs des historiens, que ce soit sur les exclus de 1789, les femmes, les esclaves un temps libérés en 1794, ou bien sûr la créativité du moment, des beaux-arts, où la Révolution n'apparait plus stérile, à la scénographie des fêtes ordonnées par le pouvoir. Ainsi s'affirment encore plus nettement l'originalité et la radicalité de la Révolution. Le thème de la "révolution atlantique" développé à partir des années 1950 - la guerre froide - comporte une observation indéniable : 1789 s'inscrit dans une vague de soulèvements de part et d'autre de l'Atlantique de 1760 à 1825 , l'indépendance des colonies espagnoles. Mais la révolution américaine n'a ni l'ambition ni la portée qu'auront les événements de France. Ceux-ci, loin de relever de la simple construction d'un a mythe national " ont une 
envergure matérielle et sociale que n'ont pas les troubles de Genève ou même d'Amsterdam. Ce sont, semble-t-il, les soldats de la République française qui ont vaincu les Provinces-Unies et non le stathouder de Hollande qui a envahi la France. Dans l'hexagone la rupture - monarchie effondrée, féodalité abolie... l'a emporté sur la continuité, même si continuité il y a bien eu également.

Dernier point : à partir de 1963-65 et plus encore après 1975 est apparue en France avec François Furet auquel Michel Vovelle se réfère fréquemment, une école d'interprétation de la Révolution qui s'est voulue à la fois nouvelle et en opposition avec la ligne dominante de Jaurès, si ce n'est Michelet, à Soboul. Il parait bien difficile de proclamer que sur le plan scientifique elle a triomphé. Elle affirme - sans théoriser vraiment - la primauté des idées dans l'évolution historique et explique la Révolution dès 1789 et surtout la dictature de l'an II par la passion égalitaire et nationaliste qui l'aurait emporté sur la scène politique avec l'intervention des masses populaires bousculant les élites éclairées, noblesse libérale et bourgeoisie cossue, qui tenaient la barre de l'Assemblée nationale. Alors qu'à l'évidence le social est une totalité où la somme est plus que le total des composantes et où les diverses instances, du démographique à l'idéologique, agissent en même temps dans le vécu des contemporains et réagissent sans cesse les unes sur les autres, cette vision qu'on peut qualifier avec Michel Vovelle d'idéaliste, au risque d'être taxé de ringardise, fractionne le tout et le réduit à des unités autonomes, si ce n'est indépendantes, les unes par rapport aux autres, parmi lesquelles le politique est en situation privilégiée. D’une dialectique de la complexité on passe à un raisonnement qui atomise la réalité et la rend peu intelligible. Par là 1793 est renvoyé vers la psychologie collective et le passéisme des foules analphabètes et superstitieuses, vue simple qui animait effectivement nombre d'hommes des Lumières. L'armée des coalisés et la pénurie économique, l'espérance aussi s'évanouissent. Cependant la critique menée par Furet, issue d'un courant qui remonte à Tocqueville et l'Orléanisme et sa peur sociale, n'a pas été inutile : elle a contraint directement ou par ricochet à mieux saisir l'étendue et le rôle de la violence, celle aussi bien de la bourgeoisic au Champ-de-Mars que celle des meurtriers de Berthier de Sauvigny ou celle de Machecoul. Elle a poussé aussi à prendre la mesure du facteur religieux.

La seconde partie du livre pose la question de l'audience et de la signification éventuelle de la Révolution dans la France d'aujourd'hui. Les célébrations du bicentenaire en 1989 ont paru marquer que la mémoire de l'événement subsistait vivement et qu'il était considéré encore comme important pour la formation de l'État-nation, voire pour l'histoire planétaire. Pour coordonner les manifestations scientifiques sur l'événement, le CNRS a mis en place une "Commission du Bicentenaire "(1983-1990). Par ailleurs de 1986 à 1990 a fonctionné la « Mission du Bicentenaire " chargée sous l'égide du gouvernement de l'organisation des cérémoniaux. Ses vicissitudes qui dépassent les incertitudes de la cohabitation de 1986-88, la mauvaise volonté dissimulée de la mairie de Paris avec Jacques Chirac, les attaques de la droite plus sur les marges - l'habituel cri du coffre-fort souffrant : "Largent des contribuables jeté par la fenêtre "-que de front, la volonté vacillante de la gauche socialiste, l'adhésion du PCF à la célébration avec son association propre "Vive 89 » et finalement des manifestations très consensuelles pour le 14 juillet et qui s'arrêtent à Valmy célébré en 1989 et non 1992 pour, dans un esprit très social-démocrate, éviter 1793 qui fâche, tout cela montre que la commémoration recelait un enjeu idéologique. Les cérémonies et plus largement les défilés, 
jeux, reconstitutions et également conférences et publications locales soutenues par les sociétés érudites et les municipalités ont illustré un vrai succès populaire, surtout en province. Malgré la pauvreté des connaissances sur l'événement révélée par les sondages de 1989 et par les travaux de P. Garcia $(1992,2000)$, il y a eu des célébrations sous des formes diverses dans $23 \%$ des communes ce qui déborde les grandes villes, et la fête parisienne avec le spectacle de J.-P. Goude fut bien accueillie. Sur le plan scientifique le succès est également là : Michel Vovelle a compté 559 colloques consacrés à la Révolution, à Paris mais aussi en province, et 146 d'entre eux ont été tenus à l'étranger, du Brésil au Japon, 116 portant aussi sur la Révolution hors de l'hexagone, ce qui manifeste le rayonnement international du fait. Sans surprise, sur la thématique la plus grande part comme pour le reste de la discipline historique revient à l'histoire politique et culturelle.

Par contre, sur d'autres plans Furet, a pu dire à juste titre « J'ai gagné ! » et répéter après les Feuillants et Bonaparte : "La Révolution est terminée ". Le concept de révolution en général a perdu son attrait et, sous l'influence en partie des événements de la fin du XX' contribué à discréditer 1789. En tant que subversion violente et radicale, les partis politiques de gauche qui étaient attachés au souvenir de la Révolution française, ont répudié la notion même de révolution. La mémoire a retravaillé le souvenir de l'événement : il cst devenu le socle fondateur de la France contemporaine suivant Michel Vovelle, mais il n'est plus envisagé comme créateur d'anticipations politiques et sociales qu'il aurait fallu établir à partir d'une lutte difficile contre les anciens dominants. Dans les médias, la conception de Furet plutôt péjorative vis-àvis de 1789 , l'a emporté. Le vieux courant d'inspiration contre-révolutionnaire, édulcoré il est vrai, connaît même depuis 1978 une véritable audience de foule qui ne se dément pas avec le spectacle son et lumière du Puy-du-Fou en Vendée. Les études sur la place de la Révolution dans les manucls d'histoire de quatrième et de seconde, les transformations imposées à la Marseillaise, admise comme hymne national même par l'extrême-droite, et l'évolution de la figure de Marianne devenue la France plutôt que la liberté et agréablement érotisée, vont dans le méme sens. La Révolution française ne suscite plus vraiment de répulsion, ni non plus d'adhésion. Elle susciterait même en nous à peu près autant d'émotion que par exemple la découverte dans ces cinquante dernières années de la civilisation du Mitanni (15001300 avant J.C.) qui ne paraît pas avoir déclenché de grandes batailles morales. En fait, davantage que l'analyse de Furet qui a subi un vieillissement brutal avec le développement d'une nouvelle historiographie des idées politiques, telle qu'clle apparaît, entre autres, dans l'Histoire des droites en France dirigée par J.-F. Sirinelli (3 vol. 1992, rééd. 2006), a agi d'abord la crise de l'État-nation et celle du régime représentatif, atteints à la fois par leur complaisance et leur faiblesse en face des entreprises transnationales, de la montée des institutions curopéennes et planétaires, et de l'éloignement technocratique du personnel politique vis-à-vis des citoyens. Plus encore l'écroulement des États du socialisme réel qui se réclamaient de l'idée révolutionnaire, a fait rejeter l'utopie. Alors, soutenu par toute une tradition de Burke à Furet, le vieil esprit fait de conservatisme social et de libéralisme d'allure moderne, né d'ailleurs avec les Thermidoriens et dessiné en France avec J.-B. Say et B. Constant, ce qui n'est plus très neuf, prônant plus ou moins ouvertement la reconnaissance de l'inégalité et de la responsabilité de l'individu juridiquement libre comme des faits intangibles et inévitables - le " pragmatisme " - a fourni la justification philosophico-historique pour que chacun, en particulier les victimes du 
système, se résigne. On a même pu faire admettre au Puy-du-Fou une partie des thèses réactionnaires en s'appuyant sur la recherche de racines effectivement détruites par la concentration capitaliste.

Pourtant, par là subsiste un autre aspect de la Révolution, des refus lors des référendums européens de 1992 et 2005, sont réapparus, selon une géographic qui rappelle les clivages anciens. Plus large que l'idéc de libération, la notion d'émancipation connaît un certain succès et avec elle reparaît l'idée, venue des Lumières et de la Révolution, de progrès, mais non pas comme un phénomène linéaire et continu, comme au contraire un mouvement doublement contradictoire : fait d'une alternance d'avancées et de reculs et, dans son contenu composé en même temps d'aspects positifs et de régressions.

Ces quelques notations à la lecture du livre suggèrent que la Révolution n'est en fait toujours pas un objet froid et vit encore, et l'un des acteurs de cette survivance est bien l'auteur, à la fois par son ouvre scientifique et par les charges qu'il a assurées à la tête de l'Institut d'Histoire de la Révolution Française de la Sorbonne, à la présidence de la Société des études robespierristes et à celle de la Commission CNRS ad hoc, en particulier dans la presse et les embúches du bicentenaire. Comme cet ouvrage en porte une preuve, chacun se souvient que ces fonctions ne consistèrent pas seulement à inaugurer les chrysanthèmes.

Guy LEMARCHAND

Lynn Hunt, Inventing Human Rights, A History, New York et Londres, W.W. Norton et Cie, 2007, 272 p., ISBN 0-393-06095-0, 25,95\$.

Lynn Hunt propose ici un ouvrage original pour parler des droits de l'homme en partant de l'expérience historique du XVIII' siècle en Europe et en Amérique du Nord et en allant jusqu'à l'usage de ces droits au $\mathrm{XX}^{\mathrm{c}}$ siècle. Elle montre les étapes par lesquelles la conscience collective s'est cmparée de cette notion jusqu'à en faire un des points essentiels de la culture occidentale. Il ne s'agit pas d'une histoire téléologique ou hagiographique, mais de la reconstitution de la démarche empirique commune à différents États, la France, la Grande-Bretagne, les États-Unis, au travers des modifications des sensibilités, pour donner à voir les conditions de l'application historique d'une notion.

Lintroduction est significative en comparant les deux versions du préambule de la Déclaration d'indépendance des États-Unis de 1776 qui passent d'une formulation marquée par le sentimentalisme de l'époque à une expression pleinement politique, instituée dans l'univers politique. Cette prise de conscience est le tournant qui fait passer la définition des droits du flou dans lequel ils se trouvent encore à la moitić du XVIII" siècle à une clarté inexorable. Cette imposition de l'évidence des droits eux-mêmes s'est réalisée par une mutation des sensibilités, via notamment la littérature romanesque. Le résultat aura été de rendre la torture judiciaire insupportable à l'opinion. Dans une approche pragmatique et pratique, Lynn Hunt insiste sur le fait que ce changement n'est pas lié à une quelconque imprégnation informelle des idées, mais qu'il s'agit bien d'une modification de la collectivité des lecteurs, convaincus par les émotions ressenties et partagées qu'ils peuvent doréna- 